Research Paper

\title{
Oxidative Stress, DNA Damage and Repair in Heart Failure Patients after Implantation of Continuous Flow Left Ventricular Assist Devices
}

\author{
Nandan Kumar Mondal ${ }^{1}$, Erik Sorensen², Nicholas Hiivala², Erika Feller ${ }^{3}$, Bartley Griffith ${ }^{1}$, and Zhongjun \\ Jon $\mathrm{Wu}^{1,}$ \\ 1. Department of Surgery, Artificial Organ Lab, University of Maryland School of Medicine, Baltimore, Maryland, United States, 21201. \\ 2. Department of Clinical Engineering, University of Maryland Medical Center, Baltimore, Maryland, United States, 21201. \\ 3. Department of Medicine, University of Maryland School of Medicine, Baltimore, Maryland, United States, 21201.
}

\begin{abstract}
$\triangle$ Corresponding author: Zhongjun Jon $\mathrm{Wu}, \mathrm{PhD}$. Artificial Organs Laboratory, Department of Surgery, University of Maryland School of Medicine, MSTF-436, 10. South Pine Street, Baltimore, MD-21201. Tel no: 410-706-7715; Fax no: 410-706-0311 Email: zwu@smail.umaryland.edu.
\end{abstract}

( ) Ivyspring International Publisher. This is an open-access article distributed under the terms of the Creative Commons License (http://creativecommons.org/ licenses/by-nc-nd/3.0/). Reproduction is permitted for personal, noncommercial use, provided that the article is in whole, unmodified, and properly cited.

Received: 2013.03.09; Accepted: 2013.05.05; Published: 2013.05.20

\begin{abstract}
Objective: To study the status of oxidative stress and DNA damage repair in circulating blood leukocytes of heart failure patients supported by continuous flow left ventricular assist devices (LVADs).

Materials and methods: Ten HF patients implanted with LVAD as bridge to transplant or destination therapy were enrolled in the study and 10 age and sex matched volunteers were recruited as the study control. Reactive oxygen species (ROS) in blood leukocytes and superoxide dismutase (SOD) in erythrocytes were measured by flow cytometry/immunofluorescence microscopy and spectrophotometry, respectively. ELISA was used to measure oxidized low density lipoproteins (oxLDL) in plasma. Markers of DNA damage $(\mathrm{y}-\mathrm{H} 2 \mathrm{AX})$ and repairs (MreII, DNA ligase IV, Ku70, and Ku80) were quantified in blood lymphocytes by immunocytochemistry.

Results: Levels of ROS and oxLDL were significantly higher in HF patients with LVAD than baseline as well as the control group; moreover, SOD levels were decreased with increasing post-operative periods. All the changes indicated enhanced oxidative stress among LVAD recipients. Significantly higher $\mathrm{Y}-\mathrm{H} 2 \mathrm{AX}$ foci in lymphocytes confirmed DNA double strand breaks in LVAD recipients. $\mathrm{Y}-\mathrm{H} 2 \mathrm{AX}$ foci numbers in lymphocytes were positively correlated with the ROS and oxLDL and negatively with SOD levels $(p<0.000$ I). Expressions of DNA ligase IV, Ku70 and Ku80 proteins were highest after one week and Mrell protein after 3 months of LVAD transplantation; indicated abnormal DNA repair.

Conclusions: The study, for the first time shows that, continuous flow LVAD implanted HF patients not only exhibit elevated oxidative stress and DNA damage in blood leukocytes but also have abnormalities in DNA repair pathways.
\end{abstract}

Key words: heart failure; left ventricular assist device; oxidative stress; DNA damage; DNA repair.

\section{Introduction}

Over the past two decades, significant evidence has suggested a close relation between pathophysi- ology of Heart failure (HF) and increased oxidative stress, both systemically and in pericardial fluid $[1,2]$. 
Oxidative stress describes an imbalance between antioxidant defences and the production of reactive oxygen species (ROS), which at high levels cause cell damage but at lower levels induce subtle changes in intracellular signalling pathways [3]. Increased oxidative stress is implicated in most types of HF, including that resulting from ischaemic and non-ischaemic cardiomyopathy, pressure and volume overload, tachycardiomyopathy and chemotherapy-induced failure [3]. The adverse effects of oxidative stress in HF patients are vascular endothelial dysfunction [4], cardiac hypertrophy [5], interstitial fibrosis [6], myocyte contractile dysfunction [7] and adverse left ventricular remodelling after myocardial infarction [2].

Heart failure (HF) is a growing epidemic with the annual number of hospitalizations constantly increasing over the last decades for HF as a primary or secondary diagnosis [8]. To address the need to support the circulation in patients with end-stage $\mathrm{HF}$ a wide variety of mechanical circulatory support devices have been developed over the past four decades. Left ventricular assist devices (LVAD) therapy has evolved into a standard therapy for patients with advanced HF $[9,10,11]$, not only as a bridge to cardiac transplantation but also as a destination therapy or a bridge to myocardial recovery $[12,13]$. This increase is attributed to the success of new second-generation continuous-flow devices [14], although a substantial portion of patients suffer from numerous co-morbidities [15].

Oxidative stress specifically activated by diverse stimuli that are important in cardiovascular pathology-for example, angiotensin II, endothelin 1, cytokines, growth factors, oxidised low density lipoproteins (oxLDL), mechanical stretch and even high shear stress [16,17]. Recently, Caruso et al., 2012 showed that the levels of oxidative-inflammatory markers were higher in LVAD recipients compared to other groups [18]. Previous investigation indicated that oxidative stress may promote the development of multiple organ failure in patients suffering from systemic inflammatory disease [19]. Moreover, LVAD is responsible for inflammatory cytokine production in HF patients and other research investigations reported that cytokine stimulation is positively associated with oxidative stress $[20,21,22]$. Oxidative stress is also responsible for damage to DNA, membranes, proteins and other macromolecules. Previous study reported that somatic DNA damage contribute to the pathogenesis of atherosclerosis and elevated levels of oxidative DNA damage in HF patients [23]. Moreover, biomarkers of oxidative DNA damage was significantly higher in HF patients and the severity of damage was positively associated with end stage HF
(NYHA class IV) [24].

The pathophysiological effects of ROS depend on the type, concentration and specific site of production. When the local levels of ROS are high, they tend to react with numerous protein centres, DNA, cell membranes and other molecules, causing considerable cellular damage as well as generating other more reactive radicals. In HF patients supported by LVADs, circulating blood is continuously experiencing non-physiological elevated shear stresses and in direct contact with the biomaterials of LVADs. Previous studies showed that high shear stress is responsible for ROS generation [25] and DNA damage [26]. So it is reasonable to assume that long-term exposure to high shear stress flow environment as well as artificial blood contacting materials in continuous flow LVADs may have an additive role in the excess production of ROS form circulating leukocytes and concomitant DNA damage in lymphocytes. However, till now there is no such report regarding oxidative stress and DNA damage in lymphocytes and the ability of repair these damage in HF patients supported by LVADs. In view of this, the present study has been conducted.

\section{Materials and Methods}

\section{Subjects}

We studied $10 \mathrm{HF}$ patients with NYHA class IV undergoing continuous flow LVAD (5 HeartMate II and 5 Jarvik 2000) implantation as bridge to transplant or destination therapy and 10 apparently healthy age and sex matched volunteers as controls. The inclusion criteria for the selection of HF patients were (i) aged between 18 to 70 years, (ii) undergoing contentious flow LVAD implantation and (iii) able to provide informed consent for the study. The inclusion criteria for the age and sex matched control subjects were (i) no clinical evidence of HF or other cardiovascular diseases, (ii) history of malignancy and (iii) inflammatory disease on careful examination and routine laboratory tests. Pregnant or breastfeeding women or women using oral contraceptives were also excluded from the study. History of tobacco use, alcoholism, diabetes and hypertension were common in HF patients in this study. The control subjects were also matched for these conditions. All procedures involving collection of human blood were approved by the Institutional Review Board (IRB). All patients and volunteers gave their written informed consent and were informed about the aims of the study in accordance with the Declaration of Helsinki.

\section{Reagents and chemicals}

A complete detail of reagents and chemicals in- 
cluding antibodies and kits used in this study can be found in Supplementary Material under Annexure I.

\section{Collection of blood sample}

EDTA-anticoagulated blood samples of the HF patients were collected before LVAD implant surgery (baseline/pre-operative: Pre-OP) and at day 7 (Post-operative duration 1 week: POD-1W), one month (POD-1M), two months (POD-2M) and three months (POD-3M) after the implant surgery. The samples were aliquoted and processed immediately according to the study protocol. Details of sample preparation can be found in Supplementary Material.

\section{Analysis of oxidative stress and antioxidant defense}

For the analysis of oxidative stress, reactive oxygen species (ROS) in blood leukocytes and superoxide dismutase (SOD) in erythrocytes were measured by flow cytometry [27, 28] / immunofluorescence microscopy and spectrophotometry, respectively. ELISA was used to measure oxidized low density lipoproteins (oxLDL) in plasma. Detailed experimental procedures can be found in Supplementary Material under Annexure II.

\section{Isolation of blood lymphocytes and immuno- fluorescence (IF) microscopy}

Detail procedures of lymphocyte isolation and IF microscopy for the assessment of markers of DNA damage $(\gamma-\mathrm{H} 2 \mathrm{AX})$ and repairs (Mre11, DNA ligase IV, $\mathrm{Ku} 70$, and Ku80) proteins can be found in Supplementary Material under Annexure III.

\section{Statistical analysis}

The results are presented as mean \pm SD (standard deviation) or median with range and statistically analyzed using SPSS statistical software (Statistical Package for Social Sciences for windows, release 10.0; SPSS Inc., Chicago, IL, USA). Statistical differences were determined by using Chi-square test, Student's t-test, Mann-Whitney $U$ test and One-Way ANOVA post hoc range tests after Bonferroni correction, as applicable. Univariate analysis was carried out using Spearman's rank correlation test to find out the relation between two measurable parameters as continuous variables, and the result was expressed as $\rho$ (rho) value. Statistical significance was assigned at $P<0.05$.

\section{Results}

\section{Demography and clinical characteristics}

Demographic and clinical characteristics of HF patients and control subjects are summarized in Table 1. HF and healthy controls were comparable with respect to age, sex, height, weight, body mass index (BMI) and body surface area. Moreover, history of smoking, alcoholism, diabetes and hypertension were also matched in both groups to exclude the effects of these conditions on the study. All of the HF patients were under NYHA class IV and etiologies of heart disease were either ischemic or non-ischemic cardiomyopathy. The last echocardiographic parameters before LVAD implantation were used for estimation of left ventricular end diastolic diameter (LVEDD) and left ventricular ejection fraction (LVEF). The HF patients were implanted with two types of LVADs, the HeartMate II LVAD and the Jarvik 2000 heart, both of which incorporate axial-flow and rotary pump technology and represent the next generation of devices.

We found Warfarin as main anticoagulant drug used by $50 \%$ HF patients. Non-steroid anti-inflammatory drug (aspirin @81 mg daily) and Cardiac glycoside (digoxin @ $125 \mu \mathrm{g}$ daily) were documented in $40 \%$ cases. Use of Beta blocker (carvedilol @ 3.125 - 12.5 mg daily), statin (atorvastatin @ 10 - $40 \mathrm{mg}$ daily) and ACE inhibitor (lisinopril @ 5 - 40 mg daily) were also noted in $70 \%, 50 \%$ and $30 \% \mathrm{HF}$ patients respectively (Table 1 ).

\section{Laboratory Hematology and Blood Chemistry}

The comparison of routine laboratory hematology and blood chemistry between the healthy controls and HF patients before and after LVAD implantation during the entire follow up period is summarized in Table 2. All the hematological and blood chemistry parameters remained within the normal range in the healthy control group while the HF patients had lower levels of hemoglobin, hematocrit and higher levels of blood urea nitrogen (BUN), creatinine and bilirubin (both total and direct) with respect to the normal range before LVAD implantation. Blood Leukocytes, erythrocytes, platelets, aspartate aminotransferase (AST) and alanine aminotransferase (ALT) of the HF patients remained within normal range but higher than those of the control group. All the differences in these tests between the HF patients and healthy controls were statistically significant $(p<0.05)$ except leukocyte count (Table 2). The HF patients supported with LVAD had significantly higher leukocyte count after one month of LVAD implantation (POD-1M) but returned almost to Pre-LVAD level at month 3 (POD-3M). Erythrocytes, hemoglobin and hematocrit values were decreased through the follow up study and remained below the normal range. Platelet count was reduced to $26 \%$ (130 \pm 95.05 vs. $175.5 \pm 75.75 \times 10^{3} / \mu \mathrm{L}$ in Pre-OP, $\mathrm{p}=0.3076$ ) at POD-1W of LVAD implantation but returned to the 
normal range at POD-1M and thereafter. BUN and creatinine levels became the highest at POD-1M but almost returned to Pre-OP levels at the end of study. Levels of total and direct bilirubin slightly increased at POD-1W of LVAD implantation but decreased at each post-operative month and returned to the normal range. AST and ALT values remained within the normal range throughout the study. However $42 \%$ ( $29 \pm 8.6$ vs. $50.3 \pm 15.2 \mathrm{U} / \mathrm{L}$ in pre-operative, $p=0.0039$ ) reduction in AST value and $41 \%$ reduction in ALT value $(28.5 \pm 15.35$ vs. $48.5 \pm 14.15 \mathrm{U} / \mathrm{L}$ in pre-operative, $\mathrm{p}=0.0169$ ) were noted at POD-3M in comparison to pre-operative values (Table 2).

\section{Oxidative stress: ROS, SOD and oxLDL}

Flow cytometric analysis showed higher MFI of DCF-DA among the HF patients when compared to the healthy controls, suggesting increased ROS gen- eration in peripheral blood leukocytes (Figure 1A-1C). The MFI of DCF-DA was $51 \%$ (678.1 \pm 131.4 vs. 448.4 $\pm 105.2), 37 \%(492.7 \pm 121.2$ vs. $358.6 \pm 111.5)$, and 54 $\%(301.6 \pm 102.3$ vs. $195.8 \pm 76.4)$ higher in peripheral blood neutrophils, lymphocytes and monocytes respectively at POD-1W of LVAD implantation when compared to the Pre-OP values. During the follow-up measurements up to 3 month after LVAD placement, the MFI of DCF-DA was significantly increased at each post-operative month and became the highest at POD-3M (Figure 1A-1C). Microscopic evaluation of intracellular ROS generation in blood leukocytes was in general agreement with the flow cytometric evaluation. The intensity of ROS positive cytoplasm of neutrophils, lymphocytes and monocytes increased with time after LVAD implantation (Figure 2).

Table I. Demographic and Clinical Characteristics.

\begin{tabular}{|c|c|c|}
\hline Characteristics & Heart failure Patients $(n=10)$ & Healthy Control subjects $(n=10)$ \\
\hline \multicolumn{3}{|l|}{ Demography } \\
\hline Age in years, median (range) & $65(57-69)$ & $63(26-74)$ \\
\hline Sex (\% male) & $80.00 \%$ & $80.00 \%$ \\
\hline Height in meter, median (range) & $1.72(1.63-1.84)$ & $1.68(1.58-1.73)$ \\
\hline Weight in kilograms, median (range) & $79.80(48.12-122.30)$ & $76.83(54.93-101.80)$ \\
\hline Body mass index $\left(\mathrm{kg} / \mathrm{m}^{2}\right)$, median (range) & $24.50(17.00-36.40)$ & $26.71(20.80-27.09)$ \\
\hline Body surface area $\left(\mathrm{m}^{2}\right)$, median (range) & $1.95(1.50-2.49)$ & $1.73(1.66-1.93)$ \\
\hline History of tobacco smoking (\%) & $70.00 \%$ & $70.00 \%$ \\
\hline History of alcohol intake (\%) & $80.00 \%$ & $80.00 \%$ \\
\hline \multicolumn{3}{|l|}{ Past medical history } \\
\hline Heart failure $(\%)$ & $100 \%$ & $0 \%$ \\
\hline Diabetes mellitus (\%) & $50 \%$ & $50 \%$ \\
\hline Hypertension $(\%)$ & $70 \%$ & $70 \%$ \\
\hline \multicolumn{3}{|l|}{ Etiology of heart disease } \\
\hline Ischemic cardiomyopathy (\%) & $50 \%$ & $0 \%$ \\
\hline Non-ischemic cardiomyopathy (\%) & $50 \%$ & $0 \%$ \\
\hline \multicolumn{3}{|l|}{ Medications on admission } \\
\hline \multicolumn{3}{|l|}{ Anticoagulants } \\
\hline Warfarin (1.0 - $7.5 \mathrm{mg}$ daily) & $50 \%$ & - \\
\hline \multicolumn{3}{|l|}{ Non-steroid anti-inflammatory drug } \\
\hline Aspirin (81 mg daily) & $40 \%$ & - \\
\hline \multicolumn{3}{|l|}{$\beta$-blockers (nonselective) } \\
\hline Carvedilol (3.125 - 12.5 mg daily) & $70 \%$ & - \\
\hline \multicolumn{3}{|l|}{ ACE inhibitor } \\
\hline Lisinopril (5 - 40 mg daily) & $30 \%$ & - \\
\hline \multicolumn{3}{|l|}{ Statins:HMG-CoA reductase inhibitors } \\
\hline Atorvastatin (10 - $40 \mathrm{mg}$ daily) & $50 \%$ & - \\
\hline \multicolumn{3}{|l|}{ Cardiac glycoside } \\
\hline Digoxin (125 $\mu$ g daily) & $40 \%$ & - \\
\hline \multicolumn{3}{|l|}{ Echocardiographic parameters } \\
\hline Left ventricular end diastolic diameter $(\mathrm{mm})$, median (range) & $68(48-77)$ & - \\
\hline Left ventricular ejection fraction, $(\%)$, median (range) & $15(10-20)$ & - \\
\hline \multicolumn{3}{|l|}{ Types of LVAD } \\
\hline HeartMate II (\%) & $50 \%$ & $0 \%$ \\
\hline Jarvik $2000(\%)$ & $50 \%$ & $0 \%$ \\
\hline
\end{tabular}


Table 2. Laboratory Hematology and blood chemistry.

\begin{tabular}{|c|c|c|c|c|c|c|c|}
\hline \multirow{3}{*}{ Variable } & \multirow{3}{*}{$\begin{array}{l}\text { Healthy } \\
\text { Control }\end{array}$} & \multirow{3}{*}{ Normal range } & \multicolumn{5}{|c|}{ Heart failure patients } \\
\hline & & & \multirow{2}{*}{$\begin{array}{l}\text { Before LVAD } \\
\text { Pre-OP }\end{array}$} & \multicolumn{4}{|l|}{ After LVAD } \\
\hline & & & & POD-1W & POD-1M & POD-2M & POD-3M \\
\hline Leukocytes $\left(\times 10^{3} / \mu \mathrm{L}\right)$ & $6.81 \pm 1.07$ & $4.5-11.0$ & $8.53 \pm 3.43$ & $11.17 \pm 2.99$ & $11.40 \pm 1.11^{\mathrm{b}}$ & $10.25 \pm 1.29$ & $8.70 \pm 2.57$ \\
\hline Erythrocytes $\left(\times 10^{6} / \mu \mathrm{L}\right)$ & $5.69 \pm 0.83$ & $4.0-5.7$ & $4.01 \pm 1.02^{\mathrm{a}}$ & $3.04 \pm 0.33^{b}$ & $3.08 \pm 0.29 \mathrm{~b}$ & $3.21 \pm 1.07$ & $3.14 \pm 0.88$ \\
\hline Platelet $\left(\times 10^{3} / \mu \mathrm{L}\right)$ & $227 \pm 52.03$ & $153-367$ & $175.50 \pm 75.74^{a}$ & $130 \pm 95.05$ & $216 \pm 87.55$ & $196.25 \pm 43.55$ & $241.50 \pm 33.33^{\mathrm{b}, \mathrm{c}}$ \\
\hline Hemoglobin (g/dL) & $15.60 \pm 0.73$ & $12.6-17.4$ & $11.26 \pm 2.79 \mathrm{a}$ & $8.97 \pm 0.88^{b}$ & $8.90 \pm 0.87 b$ & $9.38 \pm 2.90$ & $9.28 \pm 2.32$ \\
\hline Hematocrit (\%) & $45.8 \pm 2.19$ & $37-50$ & $34.75 \pm 8.42^{\mathrm{a}}$ & $26.81 \pm 2.55^{\mathrm{b}}$ & $28.00 \pm 2.71^{\mathrm{b}}$ & $30.60 \pm 9.28$ & $28.90 \pm 7.80$ \\
\hline BUN (mg/dL) & $14.76 \pm 4.57$ & $9-20$ & $30.50 \pm 11.47 \mathrm{a}$ & $29.57 \pm 21.01$ & $49.00 \pm 21.99$ & $37.50 \pm 24.97$ & $31.50 \pm 17.33$ \\
\hline Creatinine $(\mathrm{mg} / \mathrm{dL})$ & $0.84 \pm 0.31$ & $0.66-1.25$ & $1.46 \pm 0.52^{\mathrm{a}}$ & $1.59 \pm 1.25$ & $2.01 \pm 1.85$ & $1.14 \pm 0.65$ & $1.47 \pm 1.01$ \\
\hline Total bilirubin $(\mathrm{mg} / \mathrm{dL})$ & $0.34 \pm 0.11$ & $0.2-1.3$ & $2.71 \pm 1.44^{\mathrm{a}}$ & $2.78 \pm 1.93$ & $1.39 \pm 0.79 \mathrm{~b}$ & $0.77 \pm 0.30^{\mathrm{b}, \mathrm{c}}$ & $1.10 \pm 0.52^{\mathrm{b}, \mathrm{c}}$ \\
\hline Direct bilirubin $(\mathrm{mg} / \mathrm{dL})$ & $0.23 \pm 0.10$ & $\leq 0.4$ & $1.50 \pm 0.62^{\mathrm{a}}$ & $1.52 \pm 1.28$ & $1.00 \pm 0.78$ & $0.30 \pm 0.14^{\mathrm{b}, \mathrm{c}}$ & $0.35 \pm 0.07 \mathrm{~b}, \mathrm{c}$ \\
\hline $\operatorname{AST}(\mathrm{U} / \mathrm{L})$ & $31.24 \pm 8.72$ & $17-59$ & $50.33 \pm 15.20^{a}$ & $49.5 \pm 17.73$ & $50.14 \pm 19.55$ & $31.75 \pm 11.47^{b, c}$ & $29.00 \pm 8.60^{\mathrm{b}, \mathrm{c}}$ \\
\hline $\operatorname{ALT}(\mathrm{U} / \mathrm{L})$ & $27.92 \pm 13.09$ & $21-72$ & $48.50 \pm 14.15^{\mathrm{a}}$ & $34.71 \pm 22.12$ & $54.71 \pm 31.79$ & $23.50 \pm 7.85^{\mathrm{b}}$ & $28.50 \pm 15.35^{b}$ \\
\hline
\end{tabular}

BUN, blood urea nitrogen; AST, aspartate aminotransferase; ALT, alanine aminotransferase. Results are expressed as mean \pm standard deviation and p $<0.05$ is considered significant in Student's t-test. a $\mathrm{p}<0.05$ compared with control, ${ }^{b} \mathrm{p}<0.05$ compared with Pre-PO, ${ }^{c} \mathrm{p}<0.05$ compared with POD-1W
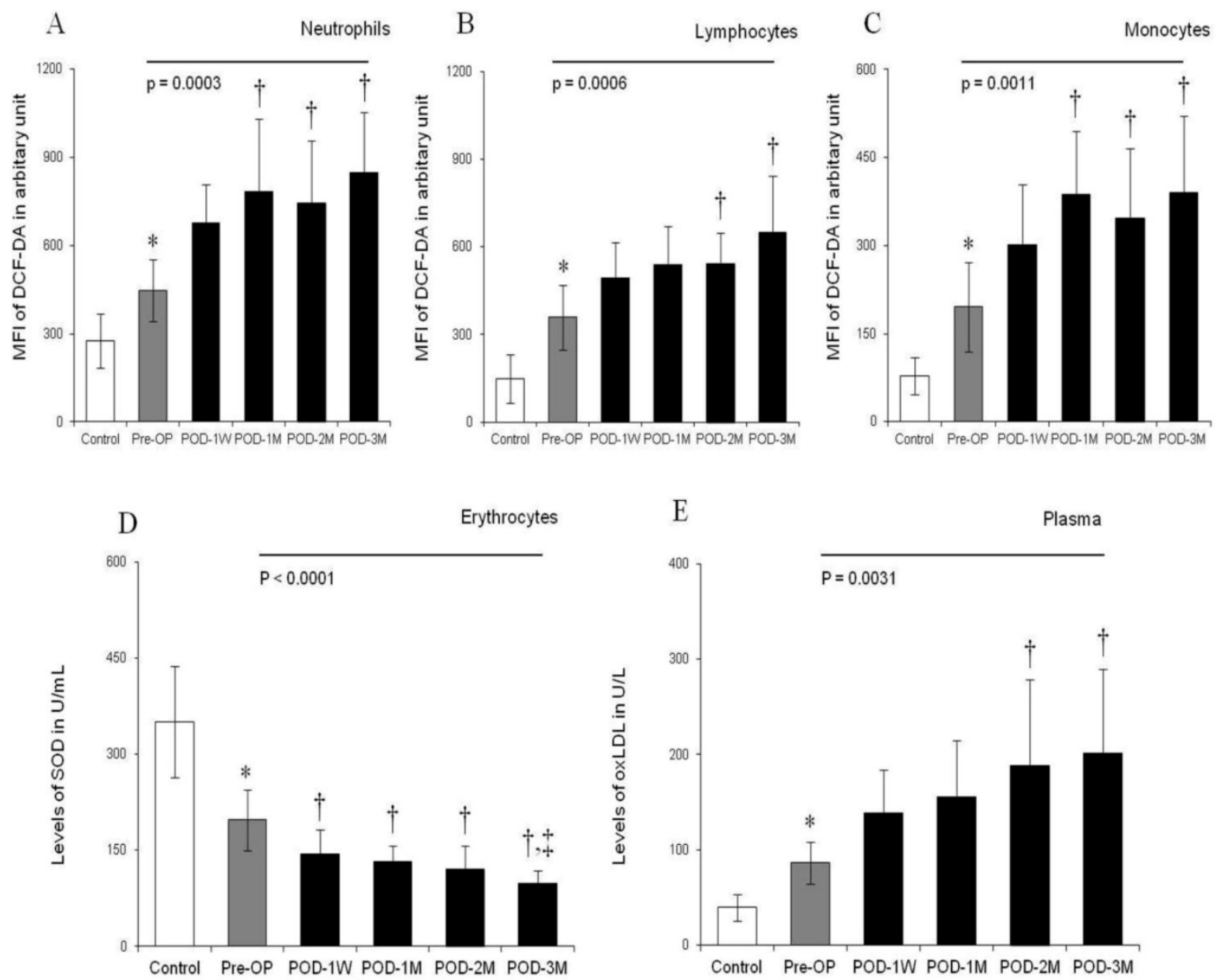

Figure I. Histograms showing levels of ROS generation (in terms of MFI of DCF-DA) in peripheral blood leukocytes [neutrophils (A), lymphocytes (B) and monocytes (C)], levels of SOD in erythrocytes (D) and levels of oxLDL in plasma (E) in control individuals, pre-operative (Pre-OP) and post-operative (POD-IW, POD-IM, POD-2M, POD-3M) heart failure patients. Bars represent standard deviation of mean. *, $\mathrm{p}<0.05$ compared with control in unpaired student's t-test, One way ANOVA for multiple comparison between Pre- and different Post-operative times found to be very significant (indicated as straight line with $p$ values). ${ }^{\dagger}, p<0.05$ compared with Pre-PO and ${ }^{\ddagger}, \mathrm{p}<0.05$ compared with POD-IW after post hoc range tests and multiple comparisons using Bonferroni corrections. 


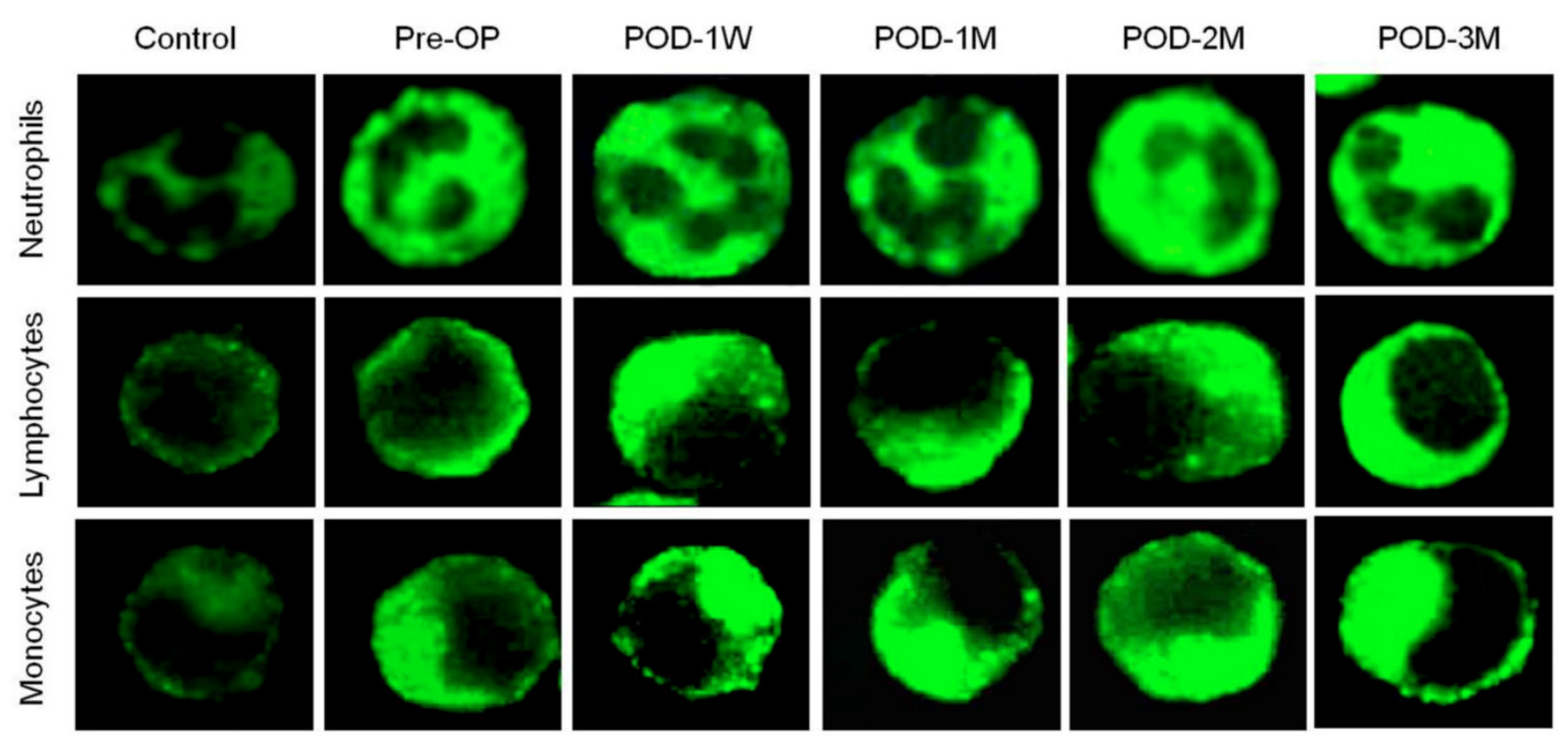

Figure 2. Photomicrograph showing ROS generation in the cytoplasm of blood neutrophils, lymphocytes and monocytes using wide-field fluorescence microscope equipped with standard green filter $(490 / 525 \mathrm{~nm})$. Note that the intensity of green fluorescence increases with increasing post-operative duration (POD-IW < POD-IM < POD-2M < POD-3M) than pre-operative condition. The fluorescence intensity remains minimum in healthy control subjects. Magnification $\times 1,000$.

A significantly lower SOD level in erythrocytes was noted in HF patients than in the healthy controls (Figure 1D). Interestingly, SOD levels progressively decreased $27 \%(144.3 \pm 38.4$ vs. $197.5 \pm 47.6 \mathrm{U} / \mathrm{mL})$, $33 \%(132.7 \pm 24.5$ vs. $197.5 \pm 47.6 \mathrm{U} / \mathrm{mL}), 38 \%$ (121.5 \pm 35.3 vs. $197.5 \pm 47.6 \mathrm{U} / \mathrm{mL})$ and $50 \%(97.8 \pm 21.3$ vs. $197.5 \pm 47.6 \mathrm{U} / \mathrm{mL})$ at POD-1W, POD-1M, POD-2M and POD-3M respectively in compared to the Pre-OP level (Figure 1D).

The study also revealed that excessive levels of plasma oxLDL, another important biomarker of oxidative stress, in the HF patients compared to the healthy control. During 3 month post-operative follow up, more than 2-fold increase in the oxLDL level was noted (Figure 1E). Spearman's rank correlation analysis showed that leukocytic ROS generation in terms of MFI of DCF-DA and plasma levels of oxLDL were positively correlated with post-operative time after LVAD implantation $(\rho=0.861, p<0.05$ and $\rho=$ $0.9783, \mathrm{p}<0.0001$ respectively) whereas there was negative correlation between concentrations of SOD in erythrocytes and post-operative periods $(\rho=$ $0.7821, \mathrm{p}<0.001$ ).

\section{DNA damage and repair}

Changes in DNA damage and repair proteins in blood lymphocytes of the healthy control subjects and the HF patients before and after LVAD implantation is presented in Table 3. To detect DNA double strand break in blood lymphocytes; $\mathrm{\gamma}-\mathrm{H} 2 \mathrm{AX}$ foci count was evaluated as each focus within nuclei represents sin- gle double strand break. Figure 3 indicates the number of foci in nuclei of blood lymphocytes. The $\mathrm{Y}-\mathrm{H} 2 \mathrm{AX}$ foci count increased progressively in post-LVAD patients when compared to pre-LVAD patients and healthy controls. The HF patients have already 2.5-fold higher DNA damage than the healthy subjects. The mean number of $\gamma-\mathrm{H} 2 \mathrm{AX}$ foci per nuclei increased by $8.4 \%(6.30 \pm 2.42$ vs $5.81 \pm 2.19$ in Pre-OP, $p=0.6776)$ in blood lymphocytes of the HF patients at POD-1W after LVAD implantation. However successive follow-up study up to 3 months shows that, the mean foci count increased by 2.5 fold ( $p=0.0007)$ to 2.7 fold ( $p=0.0008)$ at POD-2M and POD-3M compared to that that of the HF patients before LVAD implantation (Table 3).

Increased Mre-11 foci count in nuclei of blood lymphocytes indicates accelerated DNA repair either via homologous recombination (HR) or non-homologous end joining (NHEJ) pathways (Figure 4 , Table 3 ). The mean $\gamma$-H2AX foci numbers positively correlated with the post-operative time after LVAD implantation (Spearman's $\rho=0.4835, \mathrm{p}<0.01$ ). Other proteins (DNA ligase IV, Ku70 and Ku80) that help to repair double strand break exclusively via NHEJ pathway was also higher as the percent positive nuclei of blood lymphocytes significantly increased in pre-operative blood of the HF patients than the healthy control subjects. Moreover these proteins expression became highest at POD-1W after LVAD implantation and then decreased thereafter (Table 3). 
Results are expressed as mean \pm standard deviation (SD). For each subject, an average of 200 cells was screened to detect the protein expression. $p<0.05$ is considered significant in Student's t-test. a, $p<0.05$ compared with control, b, p $<0.05$ compared with Pre-PO and c, p < 0.05 compared with POD-1W

\section{Relationship between oxidative stress and DNA damage repair}

To establish a relationship between biomarkers of oxidative stress and DNA damage repair, we con- ducted Spearman's rank correlation test among LVAD recipients. Our study clearly indicated that $\mathrm{Y}-\mathrm{H} 2 \mathrm{AX}, \mathrm{Mre}-11$ and DNA ligase IV correlated positively with generation of ROS in leukocytes, in terms of MFI of DCF-DA, levels of oxLDL and negatively with concentration of SOD in erythrocytes (Table 4). The correlation between biomarkers of oxidative stress and Ku70/Ku80 was not as strong as like other damage repair biomarkers.
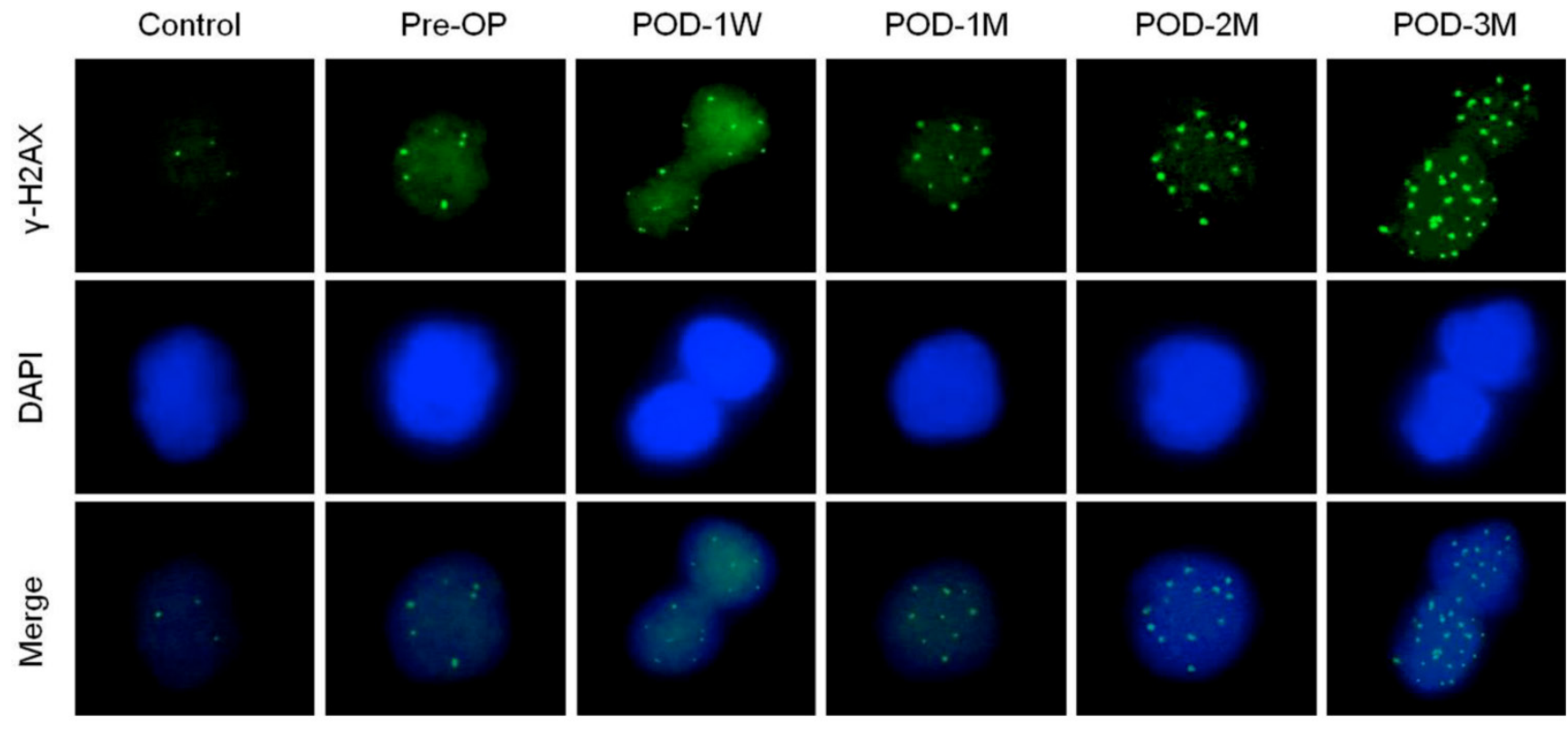

Figure 3. Immunofluorescence microscopy showing $\mathrm{y}-\mathrm{H} 2 \mathrm{AX}$ foci formation (green) in nuclei of blood lymphocytes of control, pre-operative and post-operative samples. Note highest number of $\mathrm{Y}-\mathrm{H} 2 \mathrm{AX}$ foci in nuclei of LVAD recipients after three month of implantation (POD-3M). Magnification $\times$ 1,000 .

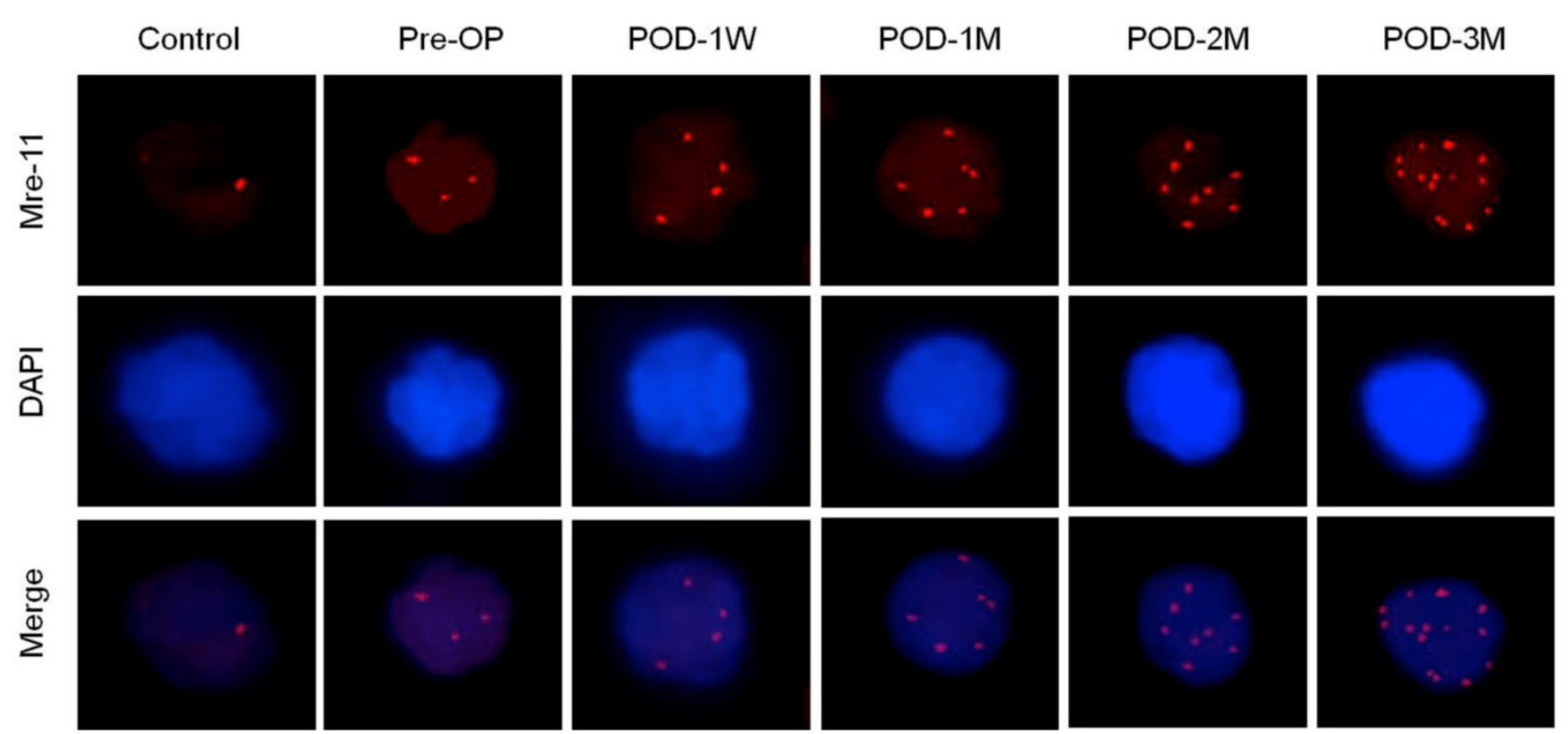

Figure 4. Immunofluorescence microscopy showing Mre-II foci formation (red) in nuclei of blood lymphocytes of control, pre-operative and post-operative samples. Note highest number of foci in nuclei of LVAD recipients after three month of implantation (POD-3M). Magnification $\times 1,000$. 
Table 3. Changes in DNA damage and repair proteins in blood lymphocytes.

\begin{tabular}{|c|c|c|c|c|c|c|}
\hline \multirow[t]{3}{*}{ Biomarkers of DNA damage / repair } & \multirow{3}{*}{$\begin{array}{l}\text { Healthy } \\
\text { Control }\end{array}$} & \multicolumn{5}{|c|}{ Heart failure patients } \\
\hline & & \multirow{2}{*}{$\begin{array}{l}\text { Before LVAD } \\
\text { Pre-OP }\end{array}$} & \multicolumn{3}{|l|}{ After LVAD } & \multirow[b]{2}{*}{ POD-3M } \\
\hline & & & POD-1W & POD-1M & POD-2M & \\
\hline$\gamma-\mathrm{H} 2 \mathrm{AX}$ foci per nucleus & $2.32 \pm 1.02$ & $5.81 \pm 2.19^{a}$ & $6.30 \pm 2.42$ & $9.83 \pm 3.61^{b, c}$ & $14.72 \pm 5.38^{b, c}$ & $15.92 \pm 6.32^{b, c}$ \\
\hline Mre-11 foci per nucleus & $1.38 \pm 0.56$ & $3.57 \pm 1.64^{\mathrm{a}}$ & $4.22 \pm 1.31$ & $6.98 \pm 1.02^{\mathrm{b}, \mathrm{c}}$ & $8.82 \pm 2.11^{b, c}$ & $11.67 \pm 3.58^{\mathrm{b}, \mathrm{c}}$ \\
\hline DNA ligase V (\% positive nuclei) & $1.77 \pm 0.23$ & $3.89 \pm 1.61^{\mathrm{a}}$ & $19.88 \pm 3.62^{b}$ & $12.32 \pm 5.29 b, c$ & $7.11 \pm 3.02^{c}$ & $8.22 \pm 3.59 c$ \\
\hline Ku70 (\% positive nuclei) & $2.11 \pm 0.58$ & $5.55 \pm 3.02^{\mathrm{a}}$ & $14.98 \pm 6.29 \mathrm{~b}$ & $9.76 \pm 3.33$ & $8.82 \pm 4.11$ & $6.87 \pm 3.11^{c}$ \\
\hline Ku80 (\% positive nuclei) & $1.98 \pm 0.43$ & $7.88 \pm 3.11^{\mathrm{a}}$ & $18.83 \pm 5.01^{b}$ & $11.08 \pm 4.77 \mathrm{c}$ & $9.91 \pm 5.10^{c}$ & $7.71 \pm 3.81^{c}$ \\
\hline
\end{tabular}

Table 4. Spearman correlation between biomarkers of oxidative stress and biomarkers of DNA damage repair in LVAD recipients.

\begin{tabular}{|c|c|c|c|c|c|c|c|c|c|c|}
\hline \multirow{3}{*}{$\begin{array}{l}\text { Biomarkers of oxi- } \\
\text { dative stress }\end{array}$} & \multicolumn{10}{|c|}{ Biomarkers of DNA damage/repair } \\
\hline & \multicolumn{2}{|c|}{$\begin{array}{l}\mathrm{\gamma}-\mathrm{H} 2 \mathrm{AX} \\
\text { foci/nucleus }\end{array}$} & \multicolumn{2}{|c|}{$\begin{array}{l}\text { Mre-11 } \\
\text { foci/nucleus }\end{array}$} & \multicolumn{2}{|c|}{$\begin{array}{l}\text { DNA ligase } \mathrm{V} \\
\text { (\% positive cells) }\end{array}$} & \multicolumn{2}{|c|}{$\begin{array}{l}\text { Ku70 } \\
\text { (\% positive cells) }\end{array}$} & \multicolumn{2}{|c|}{$\begin{array}{l}\text { Ku80 } \\
\text { (\% positive cells) }\end{array}$} \\
\hline & $\rho$-value & p-value & $\rho$-value & p-value & $\rho$-value & p-value & $\rho$-value & p-value & $\rho$-value & p-value \\
\hline ROS & 0.9429 & $<0.0001^{*}$ & 0.9427 & $<0.0001^{*}$ & 0.6145 & $0.0085^{*}$ & 0.4857 & $0.0451^{*}$ & 0.2571 & 0.3030 \\
\hline oxLDL & 0.9753 & $<0.0001^{*}$ & 0.9584 & $<0.0001^{*}$ & 0.4857 & $0.0410^{*}$ & 0.4454 & 0.0760 & 0.2354 & 0.4285 \\
\hline SOD & -0.9924 & $<0.0001^{*}$ & -0.9245 & $<0.0001^{*}$ & -0.4845 & $0.0421^{*}$ & -0.4286 & 0.0745 & -0.2125 & 0.4262 \\
\hline
\end{tabular}

Result was expressed as $\rho$ (rho) value. ${ }^{*}$, statistically significant in spearman's rank correlation test.

\section{Discussion}

Little is known about the role of continuous flow LVADs on oxidative stress and DNA damage repair among HF patients. To our knowledge, this is the first study to report on the status of oxidative stress, DNA damage and repair in end-stage heart failure patients supported by contentious-flow left ventricular assist devices. There are many experimental evidences from in vitro and animal studies that oxidative stress is common observable fact in heart failure condition [29]. Moreover, in animal models, the development of $\mathrm{HF}$ is accompanied by changes in the antioxidant defense mechanisms of the myocardium as well as elevated oxidative myocardial injury. We noticed excess ROS genetation from blood leukocytes and concomitant depletion of SOD activity in blood erythrocytes, elevated plasma levels of oxLDL, excess DNA double strand breaks and higher DNA repair proteins expression in blood lymphocytes of HF patients before LVAD implantation when compared to healthy controls. These findings suggest that oxidative stress and DNA damage in leukocytes are pre-existing conditions in HF patients. Although, we could not find any significant effects of drugs like warfarin, aspirin, digoxin carvedilol, atorvastatin and lisinopril on oxidative stress and DNA damage in pre-operative HF patients.

The causes of HF vary with age and family his- tory. In our study, most of the HF patients are older people aged between 57 - 69 years with several other health related complications. Oxidative stress has been reported to increase in elderly subjects (55 - 70 yrs. old); possibly arising from an uncontrolled production of free radicals by ageing mitochondria and decreased antioxidant defenses [30]. The alcohol intake and tobacco smoking habits were much more common in HF failure patients and the current study is not exception to these facts. Most of the HF patients were addicted to tobacco use in terms of smoking for a long period in their life history. They generally smoke 7 - 20 cigarettes a day for the last 10 to 30 years. An addiction to the consumption of alcoholic liquor was evident in $80 \% \mathrm{HF}$ patient in our study. On the other hand, half of them have history of diabetes mellitus and $70 \%$ are hypertensive. Smoking may enhance DNA damage in lymphocytes [31] and oxidative stress [32] in healthy individuals not only through the production of reactive oxygen radicals in smoke but also through weakening of the antioxidant defense systems. Elevated levels of oxidative stress and DNA damages are also evident in individuals with diabetes mellitus $[33,34]$ and hypertension $[35,36]$. The influence of these conditions to produce excess oxidative stress and DNA damage repair cannot be explained in HF patients because both control and HF groups of this study were matched (Table 1). Oxidative stress and DNA damage in the HF patients 
either suffering from Ischemic or non-ischemic cardiomyopathy was found to be similar.

In this study we also investigate the status of oxidative stress and DNA damage repair in the same HF patients after implantation of continuous flow LVAD. Interestingly, after implantation, the severity of oxidative stress and DNA damage become more prominent. Spearman's rank correlation test established a positive association between the biomarkers of oxidative stress and DNA damage after LVAD implantation. Post-operative days after implantation positively associated with increased production of ROS in blood leukocytes, plasma oxLDL levels and negatively with SOD generation in erythrocyte. Thus indicating persistent oxidative stress in these patients. Excess DNA damage after LVAD implantation is documented in terms of increased number of $\mathrm{\gamma}-\mathrm{H} 2 \mathrm{AX}$ foci count in nuclei of blood lymphocytes. This indicates most serious forms of genetic damage because each focus within nucleus represents DNA double strand break. Since both strands of the DNA double helix are broken, chromosomal fragmentation, translocation, and deletions can easily occur in these cells making them more vulnerable to genomic instability [37]. Positive association with post-implantation days was also noticed in case of DNA damage parameter. This underscores the importance of DNA repair in maintaining cellular homeostasis.

Double strand breaks (DNA damage) can be repaired either by homologous recombination (HR) or by non-homologous end joining (NHEJ) pathways. The first response of eukaryotic cells to DNA damage is the extensive phosphorylation of a member of histone H2A family like H2AX by ATM (Ataxia telangiectasia mutated) kinase. Phosphorylation occurs within 1-3 min after induction of DNA damage [38]. Rise in Mre11 foci count in nuclei of blood lymphocytes, as seen among LVAD recipients, is important in this context, because the MRN complex (Mre11, Rad50 and Nsb1) recruits ATM to the site of DNA damage where it first activates itself via autophosphorylation on Ser1981 and then phosphorylates many of its substrates including histone H2AX for initiation of DNA repair and cell cycle delay in case of cycling cells [39]. In our study, the magnitude of $\mathrm{\gamma}-\mathrm{H} 2 \mathrm{AX}$ and Mre11 foci formation in blood lymphocytes were correlated positively (Spearman's $\rho=$ 0.9984, $\mathrm{p}<0.0001$ ) during the three month follow-up study after LVAD implantation, indicating an important step in the mobilization of the DNA repair machinery at the site of DNA damage.

The human Mre11 coordinates double strand break signaling with repair by homologous recombination and is associated with the H2AX chromatin response to damage [40]. Moreover, the role of Mre11 is also implicated in non-homologous end joining (NHEJ) - a double strand break repair mechanism prevalent in non-dividing cells [41]. Since we used blood lymphocytes that are in G0 phase of cell cycle, the higher Mre11 expression can't be explained by HR pathway, rather it can help to repair DNA damage partially through DNA broken end processing via NHEJ pathway.

We also study NHEJ pathway by examining the expression of DNA ligase IV, Ku70 and Ku80 in blood lymphocyte of the HF patients before and after LVAD implantation. To repair double strand breaks via NHEJ pathway, Ku70 forms a heterodimer with Ku80 and first binds to the broken ends of the damaged DNA [42] and then DNA ligase IV ligates the DNA end to complete the repair $[42,43]$. Our results showed that these proteins were higher in nuclei of blood lymphocytes of the HF patients than the healthy controls even before LVAD implantation. Interestingly, after one week of LVAD implantation, these proteins became the highest and subsequently decreased at each month postoperatively but remained higher than the pre-operative values. These findings also tempts us to suggest that the HF patients supported by LVADs has higher levels of DNA damage in lymphocytes and subsequent activation of DNA repair pathway probably via NHEJ in which the HR protein Mre11 may plays an important role. The decreased NHEJ repair proteins after three month of LVAD implantation and the highest DNA double strand breaks at that time may pose a threat to genomic stability through damage accumulation within circulating leukocytes.

The HF patients undergoing LVAD implantation had a median body weight of 80 kilogram and after LVAD implantation we noticed significant loss of body weight especially in obese patients. Our finding is in general agreement with other reports that also showed reduction in total body weight after LVAD implantation [44,45]. Weight loss is associated with increased oxidative DNA damage not only in healthy individuals but also in people with inflammatory diseases [46]. Moreover, Mizoue et al., 2007 established a statistically significant inverse relationship between body mass index (BMI) and marker of oxidative DNA damage among healthy indivuals [47]. So, the persistent oxidative stress and DNA damage may be partly related to the decreased body weight after transplantation although the role of continuous flow LVAD cannot be underestimated.

One potential limitation of continuous-flow pump technology is the relatively high nonphysiological shear stress imparted on blood components as 
they move through the device [48]. Using computational fluid dynamics study we recently showed that continuous flow LVAD generates high shear stresses especially from axial VADs compared to centrifugal VADs [49]. Generation of reactive oxygen species and DNA damage is also related to high shear stress $[25,26]$. Thus long-term LVAD support may have an additive role in the excess production of ROS form circulating leukocytes and concomitant DNA damage in lymphocytes.

Complications during LVAD support, such as thromboembolism, bleeding, infections, and right ventricular failure remained an issue for long-term LVAD use. While staying in the intensive care units (ICUs) after LVAD implantation, patients encountered several clinical complications. Cardiogenic shock, pulmonary insufficiency following trauma or surgery, abnormal coagulation profile, anemia due to acute blood loss, thrombocytopenia, driveline infections, acute respiratory failure, acute renal failure, dysphagia and hyperglycemia were common during first two week of ICU stay. Non-surgical gastrointestinal bleeding events and right ventricular failure were noticed in $30 \%$ HF patients within one month of LVAD implantation. Pseudomonas infection was found in only two HF patients after implantation. This study indicated another important challenge of oxidative stress and concomitant DNA damage and repair after LVAD implantation in long-term basis. Besides genetic damage [28], long-term oxidative stress may be directly or indirectly related to several consequences like inflammations [50,51], bacterial infections [52], respiratory disease [53], gastrointestinal bleeding [54] and even may promote the development of multiple organ failure [19].Thus continued improvement of LVAD technology for less traumatic devices and understanding of device-induced ROS and DNA damage continue to be important.

\section{Study limitation}

We acknowledge that our study has some limitations. This study was conducted in a single center, and consequently included a limited number of end-stage HF patients undergoing continuous flow LVAD implantation. Pre-implant HF patients were compared with matched healthy controls rather than comparative group of control patients with advanced HF, say INTERMACS 1,2 and 3 . We also could not explore and show any causal relationship between LVAD parameter and ROS mediated DNA damage in HF patients after implantation. Hence, it is premature to comment on a possible direct causal relationship between LVAD parameters and subsequent ROS generation and DNA damage repair. Moreover we could not study the effects of medications used during the LVAD support although we mentioned the documentation medications on admission before LVAD implantation. Our current study has only $20 \%$ HF patients who were not addicted to alcoholic liquor consumption. So the relevance to more general (non-alcoholic) heart failure population is limited. Despite all these limitations, our study clearly indicated persistent oxidative stress, DNA damage and altered DNA repair in circulating blood cells after LVAD support. Further detailed investigations are necessary to find out the exact cause of oxidative stress and DNA damage repair in LVAD implanted HF patients.

\section{Supplementary Material}

Annexure I, II, and III. http://www.medsci.org/v10p0883s1.pdf

\section{Acknowledgements}

This work was supported by a grant from the National Institutes of Health (NIH) to Dr. Zhongjun J Wu (R01 HL 088100).

\section{Competing Interests}

The authors have declared that no competing interest exists.

\section{References}

1. Mallat Z, Philip I, Lebret M, et al. Elevated levels of 8-iso-prostaglandin F2 $\alpha$ in pericardial fluid of patients with heart failure: a potential role for in vivo oxidant stress in ventricular dilatation and progression to heart failure. Circulation. 1998; 97: 1536-9.

2. Giordano F J. Oxygen, oxidative stress, hypoxia, and heart failure. J Clin Invest. 2005; 115: 500-8.

3. Seddon M, Looi YH, Shah AM. Oxidative stress and redox signalling in cardiac hypertrophy and heart failure. Heart. 2007; 93: 903-7.

4. Dixon LJ, Morgan DR, Hughes SM, et al. Functional consequences of endothelial nitric oxide synthase uncoupling in congestive cardiac failure. Circulation. 2003; 107: 1725-8.

5. Cave AC, Grieve D J, Johar S. et al. NADPH oxidase-derived reactive oxygen species in cardiac pathophysiology. Philos Trans R Soc. 2005; 360: 2327-34.

6. Tsutsui H, Ide T, Kinugawa S. Mitochondrial oxidative stress, DNA damage, and heart failure. Antioxid Redox Signal. 2006; 8: 1737-44.

7. Gao WD, Liu Y, Marban E. Selective effects of oxygen free radicals on excitation-contraction coupling in ventricular muscle. Implications for the mechanism of stunned myocardium. Circulation. 1996; 94: 2597-604.

8. Giamouzis G, Mastrogiannis D, Koutrakis K, et al. Telemonitoring in chronic heart failure: a systematic review. Cardiol Res Pract. 2012; 2012:410820. doi: 10.1155/2012/410820.

9. Goldstein DJ, Oz MC, Rose EA. Implantable left ventricular assist devices. N Engl J Med. 1998; 339: 1522-33.

10. Rose EA, Gelijns AC, Moskowitz AJ, et al. Randomized Evaluation of Mechanical Assistance for the Treatment of Congestive Heart Failure (REMATCH) Study Group. Long-term use of a left ventricular assist device for end-stage heart failure. N Engl J Med. 2001; 345: 1435-43.

11. Slaughter MS, Rogers JG, Milano CA, et al. HeartMate II Investigators. Advanced heart failure treated with continuous-flow left ventricular assist device. N Engl J Med. 2009; 361: 2241-51.

12. Birks EJ, Yacoub MH, Banner NR, et al. The role of bridge to transplantation: should LVAD patients be transplanted? Curr Opin Cardiol. 2004; 19: $148-53$. 
13. Birks EJ, Tansley PD, Hardy J, et al. Left ventricular assist device and drug therapy for the reversal of heart failure. N Engl J Med. 2006; 355: 1873-84.

14. Slaughter MS, Pagani FD, Rogers JG, et al. HeartMate II Clinical Investigators. Clinical management of continuous-flow left ventricular assist devices in advanced heart failure. J Heart Lung Transplant. 2010; 29 (4 Suppl): S1-39.

15. Jeevanandam V. The evolution of cardiac assist device technology. J Heart Lung Transplant. 2011; 29: 11-2.

16. Li JM, Shah AM. Endothelial cell superoxide generation: regulation and relevance for cardiovascular pathophysiology. Am J Physiol Regul Integr Comp Physiol. 2004; 287: R1014-30.

17. Lambeth JD. NOX enzymes and the biology of reactive oxygen. Nat Rev Immunol. 2004; 4: 181-9.

18. Caruso R, Verde A, Campolo J, et al. Severity of oxidative stress and inflammatory activation in end-stage heart failure patients are unaltered after 1 month of left ventricular mechanical assistance. Cytokine. 2012; 59: 138-44.

19. Motoyama T, Okamoto K, Kukita I, et al. Possible role of increased oxidant stress in multiple organ failure after systemic inflammatory response syndrome. Crit Care Med. 2003; 31: 1048-52.

20. Singal BK, Beamish RE, Dhalla NS. Potential oxidative pathways of catecholamines in the formation of lipid peroxides and genesis of heart disease. Adv Exp Med Biol. 1983; 161: 391-401.

21. O'Donnell VB, Spycher S, Azzi A. Involvement of oxidants and oxidantgenerating enzyme(s) in tumour necrosis factor-a-mediated apoptosis: role for lipoxygenase pathway but not mitochondrial respiratory chain. Biochem J. 1995; 310: 133-41.

22. Cortopassi G, Wang E. Modelling the effects of age-related mtDNA mutation accumulation: complex I deficiency, superoxide and cell death. Biochim Biophys Acta. 1995; 1271: 171-6.

23. Botto N, Masetti S, Petrozzi L, et al. Elevated levels of oxidative DNA damage in patients with coronary artery disease. Coron Artery Dis. 2002; 13: 269-74.

24. Kobayashi S, Susa T, Tanaka $\mathrm{T}$, et al. Urinary 8-hydroxy-2'-deoxyguanosine reflects symptomatic status and severity of systolic dysfunction in patients with chronic heart failure. Eur J Heart Fail. 2011; 13: 29-36.

25. Noguchi N. Role of oxidative stress in adaptive responses in special reference to atherogenesis. J Clin Biochem Nutr. 2008; 43: 131-8.

26. Triyoso DH, Good TA. Pulsatile shear stress leads to DNA fragmentation in human SH-SY5Y neuroblastoma cell line. J Physiol. 1999; 515: 355-65.

27. Rothe G, Valet G. Flow cytometric analysis of respiratory burst activity in phagocytes with hydroethidine and 2, 7-dichlorofluorescein. J Leukoc Biol. 1990; 47: 440-8.

28. Mondal NK, Mukherjee B, Das D, et al. Micronucleus formation, DNA damage and repair in premenopausal women chronically exposed to high level of indoor air pollution from biomass fuel use in rural India. Mutat Res. 2010; 697: 47-54.

29. Mak S, Newton GE. The oxidative stress hypothesis of congestive heart failure: radical thoughts. Chest. 2001; 120: 2035-46.

30. Andriollo-Sanchez M, Hininger-Favier I, Meunier N, et al. Age-related oxidative stress and antioxidant parameters in middle-aged and older European subjects: the ZENITH study. Eur J Clin Nutr. 2005; 59 (Suppl 2): S58-62.

31. Alsatari ES, Azab M, Khabour OF, et al. Assessment of DNA damage using chromosomal aberrations assay in lymphocytes of waterpipe smokers. Int J Occup Med Environ Health. 2012; 25: 218-24.

32. Isik B, Ceylan A, Isik R. Oxidative stress in smokers and non-smokers. Inhal Toxicol. 2007; 19: 767-9.

33. Yang H, Jin X, Kei Lam CW, et al. Oxidative stress and diabetes mellitus. Clin Chem Lab Med. 2011; 49: 1773-82.

34. Simone S, Gorin Y, Velagapudi C, et al. Mechanism of oxidative DNA damage in diabetes: tuberin inactivation and downregulation of DNA repair enzyme 8-oxo-7,8-dihydro-2'-deoxyguanosine-DNA glycosylase. Diabetes. 2008; 57: 2626-36.

35. Ceriello A. Possible role of oxidative stress in the pathogenesis of hypertension. Diabetes Care. 2008; 31 (Suppl 1): S181-4.

36. Negishi H, Ikeda K, Kuga S, et al. The relation of oxidative DNA damage to hypertension and other cardiovascular risk factors in Tanzania. J Hypertens. 2001; 19: 529-33.

37. Mills KD, Ferguson DO, Alt FW. The role of DNA breaks in genomic instability and tumorigenesis. Immunol Rev. 2003; 194: 77-95.

38. Houtgraff JH, Versmissen J, van der Giessen WJ. A concise review of DNA damage checkpoints and repair in mammalian cells. Cardiovasc Revasc Med. 2006; 7: 165-72.
39. Kitagawa R, Bakkenist CJ, McKinnon PJ, et al. Phosphorylation of SMC1 is a critical downstream event in the ATM-NBS1-BRCA1 pathway. Genes Dev. 2004; 18: 1423-38.

40. Xie A, Kwok A, Scully R. Role of mammalian Mre11 in classical and alternative nonhomologous end joining. Nat Struct Mol Biol. 2009; 16: 814-8.

41. Adelman CA, Petrini JH. Division of labor: DNA repair and the cell cycle specific functions of the Mre11 complex. Cell Cycle. 2009; 8: 1510-4.

42. Baumann P, West SC. DNA end joining catalyzed by human cell-free extracts. Proc Natl Acad Sci U SA. 1998; 95: 14066-70.

43. Hefferin ML, Tomkinson AE. Mechanism of DNA double-strand break repair by non-homologous end joining. DNA Repair. 2005; 4: 639-48.

44. Clark AL, Loebe M, Potapov EV, et al. Ventricular assist device in severe heart failure: effects on cytokines, complement and body weight. Eur Heart J. 2001; 22: 2275-83.

45. Dhesi P, Simsir SA, Daneshvar D, et al. Left ventricular assist device as 'bridge to weight loss' prior to transplantation in obese patients with advanced heart failure. Ann Transplant. 2011; 16: 5-13.

46. Kouda K, Tanaka T, Kouda M, et al. Low-energy diet in atopic dermatitis patients: clinical findings and DNA damage. J Physiol Anthropol Appl Human Sci. 2000; 19: 225-8.

47. Mizoue T, Tokunaga S, Kasai $\mathrm{H}$, et al. Body mass index and oxidative DNA damage: a longitudinal study. Cancer Sci. 2007; 98: 1254-8.

48. Suarez J, Patel CB, Felker GM, et al. Mechanisms of bleeding and approach to patients with axial-flow left ventricular assist devices. Circ Heart Fail. 2011; 4: 779-84.

49. Fraser $\mathrm{KH}$, Zhang $\mathrm{T}$, Taskin ME, et al. A quantitative comparison of mechanical blood damage parameters in rotary ventricular assist devices: shear stress, exposure time and hemolysis index. J Biomech Eng. 2012; 134: 081002. doi: 10.1115/1.4007092.

50. Hakim J. Reactive oxygen species and inflammation. C R Seances Soc Biol Fil. 1993; 187: 286-95.

51. Banerjee A, Mondal NK, Das D, et al. Neutrophilic inflammatory response and oxidative stress in premenopausal women chronically exposed to indoor air pollution from biomass burning. Inflammation. 2012; 35: 671-83.

52. Sun $\mathrm{G}, \mathrm{Xu} X$, Wang $\mathrm{Y}$, et al. Mycoplasma pneumoniae infection induces reactive oxygen species and DNA damage in A549 human lung carcinoma cells. Infect Immun. 2008; 76: 4405-13.

53. Ryrfeldt A, Bannenberg G, Moldeus P. Free radicals and lung disease. Br Med Bull. 1993; 49: 588-603.

54. Otamiri T, Sjodahl R. Oxygen radicals: their role in selected gastrointestinal disorders. Dig Dis Sci. 1991; 9: 133-41. 